\title{
Validation of Minimum Electron Beam Dosage on Characterization of Carbon- Depletion in ULK Dielectric Materials by EELS Quantifications
}

\author{
Wayne Zhao $^{1}$ and Michael Gribelyuk ${ }^{2}$ \\ 1. Center for Complex Analyses, GLOBALFOUNDRIES, Malta, New York, USA. \\ 2. Advanced Technology Development, GLOBALFOUNDRIES, Malta, New York, USA.
}

Various advanced back-end-of-line (BEOL) integration schemes have been applied into high volume manufacturing (HVM) of Si-based semiconductor devices. Copper-based interconnects with ultra low-k (ULK) dielectric represent the current industry standard to enhance electronic device performance and reliability. $\mathrm{SiOC}(\mathrm{H}) \mathrm{ULK}$ dielectric is extremely sensitive to the electron beam illumination due to porosity of the film [1]. Over-exposure of the ULK in the electron microscope can directly lead to carbon-depletion, which alters the nature of the ULK being analyzed. Therefore physical failure analysis (PFA) of such beam-sensitive materials with the least possible electron damage presents a challenge [14]. Reliable advanced characterization methods of these nanometer scaled ULKs in BEOL are beneficial to technology node development, yield learning, and yield enhancement for HVM [2-4].

Here we extend our earlier investigations on how to minimize the electron beam damage during the carbon depletion study in the ULK dielectrics. Initially we had shown that STEM EELS by Digiscan Spectrum Imaging as the best acquisition technique, after having compared the total electron flux from the same field of view against various Analytical TEM methods, such as mapping by EFTEM, STEM EELS by TIA [2-4]. Later we had fine-tuned the instrument setup within the STEM EELS elemental mapping by Digiscan Spectrum Imaging, through a series of analyses whereby the acquisition time at each pixel was the only factor being varied. As reported earlier [2], we had successfully identified the best imaging / acquisition parameters with the minimum electron beam dose / flux, which introduced the least electron beam damage to the ULK. In [2], individual elemental line profiles of Carbon along with $\mathrm{Si}$ and $\mathrm{O}$ were extracted from each analysis and compared. As demonstrated in the line-profiles displayed in Fig. 1a, Carbon distribution becomes more uniform, and the ULK structure appears less and less grainy in STEM image as beam exposure decreases. Evidently, the shortest pixel time of 4.5 millisecond yields the smoothest Carbon distribution in contrast to more noisy profiles, which were acquired with $\mathrm{t}=90$ millisecond (and above) acquisition time. This indicates that, the shortest exposure indeed leads to the least electron beam damage of the original ULK structure.

To further enhance the confidence level in effectiveness of the approach, here we present quantitative analysis of the Carbon/Oxygen ratios as dependent on the electron beam exposure. Specifically, C/O relative compositional ratio maps were extracted from data acquired by Spectrum Imaging. As evident in Fig. $1 \mathrm{~b}$ and Fig. 1c, $\mathrm{C} / \mathrm{O}$ ratio profiles acquired with the shortest pixel time, 4.5 millisecond, preserved the highest level of carbon retention in the ULK. That is, the least electron flux induced the minimum Carbon depletion. Figure-2 is an application of this technique in a real world PFA case of analysis of the ULK, where reasonably "FLAT" C profile from raw data of EELS quantification relative compositional ratio mapping was accomplished. It should be pointed out that, the current experiments were carried out using the $200 \mathrm{kV}$ TEM instrument. Further exploration regarding the effect of the beam damage by lower acceleration voltages is still necessary [8]. 


\section{References:}

[1] Maex K. et al, J. Appl. Phys. 93, (2003), p. 8793.

[2] W. Zhao, M. Gribelyuk, Microscopy \& Microanalysis, 2(S3), (2016), p. 334.

[3] W. Zhao, M. Gribelyuk, et al., Microscopy \& Microanalysis, 21(S3), (2015), p. 1036.

[4] W. Zhao, et al, Microscopy \& Microanalysis, 20(S3), (2014), p. 362.

[5] R. Leapman and J. Hunt, Microscopy, Microanalysis, Microstructure, 2 (1991) p. 231.

[6] H. Harrach, et al, Microscopy \& Microanalysis, 16(S2), (2010), p.1312.

[7] http://www.gatan.com/products/sem-imaging-spectroscopy/digiscan-ii-system.

[8] Authors would like to express sincere thanks to Cynthia Martin and Nikolaus Schad for their excellence in TEM-preparations. Thanks also go to Fab8 Characterization Management and Legal team for supports in the publication clearance.
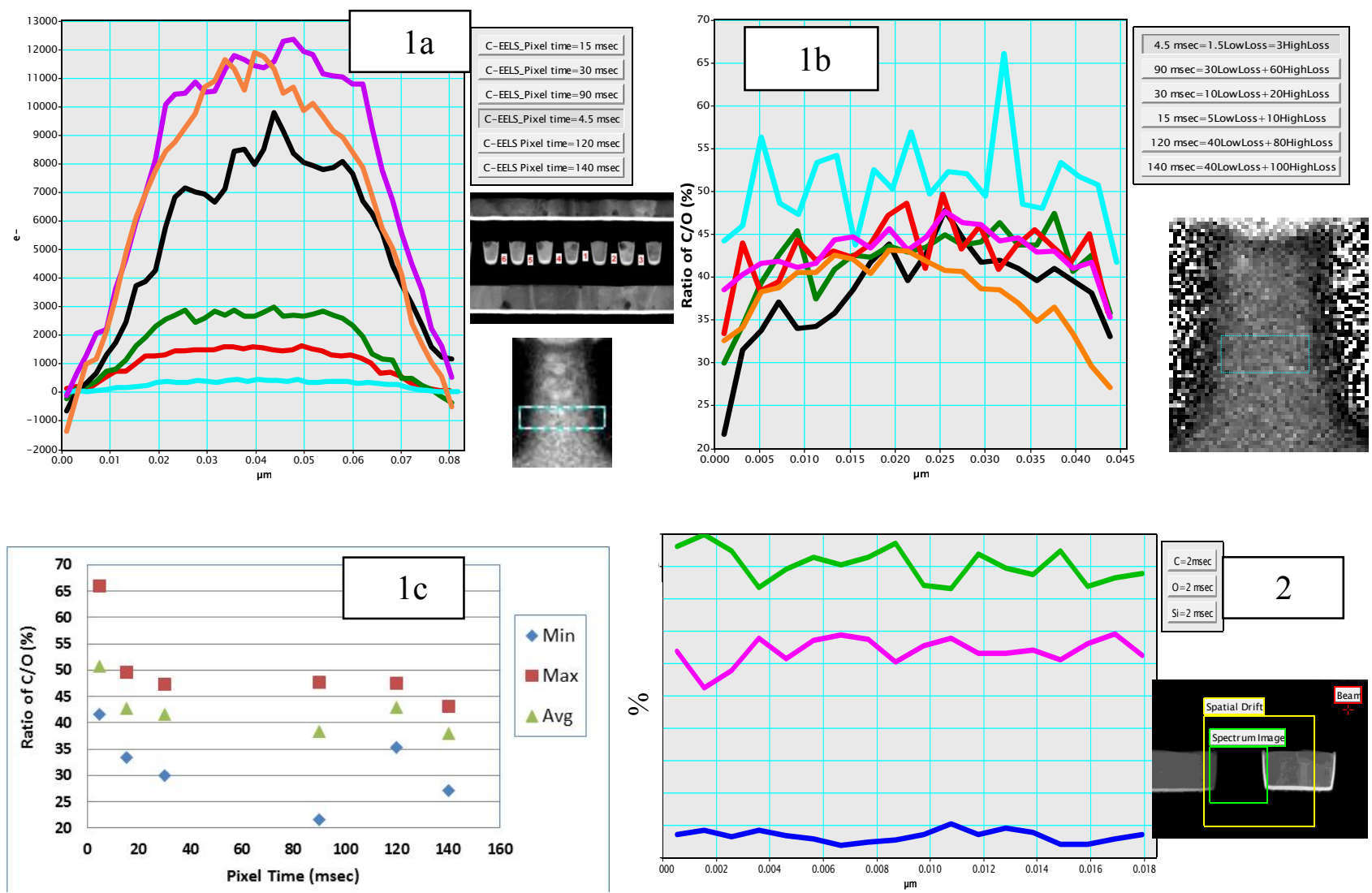

Figure 1. (1a) Overlay of extracted Carbon profiles from EELS elemental maps, and (1b) retention of C by $\mathrm{C} / \mathrm{O}$ relative compositional ratio mapping profiles, and its range display in (1c), with Digiscan dual EELS spectrum imaging total pixel time, ranging from 4.5 to 140 milli-second.

Figure 2. Application of the least beam dosage technique in one ULK characterization, with EELS quantification relative ratio mapping for reasonably flat profiles of Si-L, and C-K, and O-K, acquired by a Single EELS, with 2 milli-second pixel time, following the guideline in Fig. 1a 3c. 